\title{
MiR-384 inhibits the proliferation of colorectal cancer by targeting AKT3
}

\author{
Yong-Xia Wang, Hui-Fang Zhu, Zhe-Ying Zhang, Feng Ren and Yu-Han Hu*
}

\begin{abstract}
Background: Growing evidence suggests that MiRNAs play essential roles in the initiation and progression of colorectal cancer (CRC). The aberrant expression of miR-384 has been reported in some cancers. However, the role and mechanism of miR-384 in CRC proliferation remains unknown.

Methods: The expression of miR-384 was detected in CRC and their paired normal tissues by real-time PCR. In vivo and in vitro assays were conducted to confirm the role of miR-384 in the proliferation of CRC. Bioinformatics analysis, luciferase reporter assays, western blot and in vitro assays were used to confirm that AKT3 was the target gene of miR384. Finally, Spearman's correlation analyses was carried out to analyze the relationship between miR-384 expression and AKT3 expression in CRC.
\end{abstract}

Results: MiR-384 was down-regulated in CRC tissues. The in vivo and vitro functional assays verified that the ectopic upregulation of miR-384 inhibited the proliferation of CRC and the inhibition of miR-384 promoted the proliferation of CRC. Bioinformatics analysis, luciferase reporter assays, western blot and in vitro functional assays confirmed AKT3 as the target gene of miR-384. The expression of miR-384 was negatively correlated with the expressions of AKT3.

Conclusion: Our study verified that miR-384 could significantly suppress the proliferation of CRC by directing targeting AKT3.

Keywords: Colorectal cancer, MiR-384, AKT3, Proliferation

\section{Background}

Colorectal cancer (CRC) is one of the most common malignant tumors worldwide with high morbidity and mortality [1]. In the past 10 years, the incidence and mortality of CRC has increased rapidly in China $[2,3]$. The initiation of CRC is a complicated process which includes the activation of oncogenes, the inactivation of tumor suppressor genes and multiple risk factors $[4,5]$. Numerous genetic alterations, such as the microsatellite instability, PIK3CA, RAS and BRAF mutations, have been recognized to be involved in the tumorigenesis of CRC $[6,7]$. However, other genetic and epigenetic alterations responsible for this disease remain largely unknown. Therefore, in-depth study of the molecular mechanism in the initiation and progression of CRC is significant for

*Correspondence: hyhms@126.com

Department of Pathology, School of Basic Medical Sciences, Xinxiang Medical University, Xinxiang 453003, China exploring novel molecular targets for CRC prevention and treatment.

MicroRNAs (miRNAs) are small non-coding RNA molecules which are highly conserved in evolution and participate in the regulation of gene expression by directly being bound to the $3^{\prime}$-untranslated region (3'-UTR) of their target mRNAs $[8,9]$. It has been reported that miRNAs play crucial roles in the genesis and development of human cancer [10-12]. Recent studies have showed that miRNAs might be novel biomarkers for CRC. For example, MiR-215-5p expression is down-regulated in CRC [13]. MicroRNA-338-3p is down-regulated in thyroid cancer tissues and inhibits the progression of thyroid cancer by repressing AKT3 expression [14].

MiR-384 has been demonstrated to repress the proliferation and metastasis of pancreatic cancer [15]. Also, it has been indicated that miR-384 could inhibit CRC metastasis by directly targeting KRAS and CDC42 in our previous study [16]. The number of metastatic nodules in 
miR-384 over-expressed SW480 cells increased with the restoration of KRAS and CDC42. But the volume of the metastatic nodules could not be restored accordingly. The results suggested that miR-384 might regulate the proliferation of CRC not by targeting KRAS or CDC42. Therefore, in the current study, we will focus on delineating the role and mechanism of miR-384 in CRC proliferation.

\section{Methods}

\section{Tissue specimens and cell culture}

The fresh CRC and the matched normal colorectal tissues were collected from the Department of Pathology, Third Affiliated Hospital of Xinxiang Medical University (Xinxiang, China) from September 2016 to December 2017. All patients did not receive chemotherapy, radiotherapy or immunotherapy prior to surgery. All tissues were freshly frozen in liquid nitrogen until further use. All the cases had been diagnosed with adenocarcinoma on the basis of hematoxylin-eosin (HE) staining. The prior approval for the study had been obtained from Xinxiang Medical University Institutional Board (Xinxiang, China).

The stable cells of SW480/miR-384, SW480/Vector, HCT116/miR-384, HCT116/Vector, and LOVO/ miR-384-in, LOVO/NC, SW620/miR-384-in, SW620/ $\mathrm{NC}$ established in our previous study were cultured in RPMI-1640 or DMEM (Invitrogen). The cells of SW480, HCT116, LOVO and SW620 were obtained from American Type Culture Collection (ATCC). The medium was supplemented with $10 \%$ fetal bovine serum(FBS, Gibco) and $1 \%$ penicillin/streptomycin (Invitrogen).

RNA isolation, reverse transcription (RT) and real-time PCR Following the manufacturer's instruction, the total RNA was extracted from the cultured cells and fresh CRC tissues with Trizol (Invitrogen, USA). $2 \mu \mathrm{g}$ of total RNA synthesized the cDNA. Quantification of miR-384 was conducted by the All-in-One TM miRNA real-time PCR Detection Kit (GeneCopoeia, China) via the Applied Biosystems 7500 Sequence Detection system mixed with 5 ng cDNA and $10 \mathrm{pM}$ of each primer. The cycling conditions were conducted as previously described [16]. As for the target gene of AKT3, the primers were shown in Additional file 1: Table S1. The data were normalized to U6 or GAPDH and calculated as $2^{-\Delta \Delta C T}$.

\section{Western blot}

Protein lysates obtained from the cells were subjected to SDS-PAGE and then the gel was transferred to Polyvinylidene difluoride (PVDF, Merck Millipore). The PVDF membranes were blocked with $5 \%$ non-fat dry milk and then the membranes were incubated with rabbit antiAKT3 (1:200, Proteintech, USA) or mouse anti- $\alpha$-tubulin (1:2000, Proteintech, USA) overnight at $4{ }^{\circ} \mathrm{C}$. The next day, they were incubated with the appropriate secondary antibodies (HRP-conjugated anti-rabbit IgG (1:5000, CST, USA) or HRP-conjugated anti-mouse IgG (1:5000, CST,USA) and detected with a chemiluminescence imaging analysis system (Tanon, China).

\section{MTT assay}

$1 \times 10^{3}$ cells were seeded on 96-well plates and cultured for 24 h. $20 \mu \mathrm{l} 5 \mathrm{~g} / \mathrm{l}$ 3-(4,5-dimethylthiazol-z-yl)-2,5-diphenyltetrazolium bromide (MTT, Sigma, USA) was added to each well and incubated for $4 \mathrm{~h}$. Then, MTT was removed and $150 \mu$ dimethyl sulphoxide (DMSO; Sigma, USA) was added to the wells. The absorbance was measured at $450 \mathrm{~nm}$ with a microplate autoreader (Bio-Rad, Hercules, CA, USA). The experiment was conducted repeatedly for three times.

\section{Colony formation assay}

The cells were trypsinized and plated on 6-well plates (200 cells/well) and cultured for 2 weeks. The colonies were stained with Hematoxylin for 30 min after fixation with $4 \%$ paraformaldehyde for $10 \mathrm{~min}$. The number of colonies, defined as $>50$ cells/colony, was counted. Three independent experiments were performed.

\section{Soft agar assay}

Six-well plates were covered with a layer of $0.6 \%$ agar (Sigma, USA) in medium supplemented with $20 \%$ fetal bovine serum. Cells were prepared in $0.3 \%$ agar and seeded in triplicate at 3 a dilution of $1 \times 10^{3}$. The plates were incubated at $37{ }^{\circ} \mathrm{C}$ in a humid atmosphere of $5 \%$ $\mathrm{CO}_{2}$ for 2 weeks. Each experiment was repeated at least 3 times. Colonies were photographed after 2 weeks at an original magnification of $200 \times$.

\section{Tumorigenesis in nude mice}

4 to 6-week-old BABL/c nude mice were purchased from the Center of Laboratory Animal Science of Guangdong (Guangzhou, China). All animal experiments were conducted in accordance with current Chinese regulations and standards regarding the use of laboratory animals, and all animal procedures were approved by the Xinxiang Medical University Institutional Animal Care and Use Committee. Xenograft tumors were generated by subcutaneous injection of $2 \times 10^{6}$ stable cells of SW480/ Vector, SW480/miR-384, LOVO/NC, and LOVO/miR384-in $(n=6)$ on the hindlimbs. Another 6 nude mice were used to conduct the restoration experiments in vivo. 3 weeks later, all mice were euthanized by dislocating the cervical spine. Tumor size was measured by a slide caliper (volume $=$ length $\times$ width $\times$ height). The tumors were fixed with $10 \%$ neutral buffered formalin and embedded in paraffin. Then $4 \mu \mathrm{m}$ sections were cut and stained with 
haematoxylin and eosin according to standard protocols. Sections further underwent immunohistochemistry (IHC) staining: They were sections baked at $60{ }^{\circ} \mathrm{C}$ for $1 \mathrm{~h}$, deparaffinized with xylenes and rehydrated with graded ethanol. After incubation in $3 \% \mathrm{H}_{2} \mathrm{O}_{2}$ to quench the endogenous peroxidase activity, the sections were heated in $0.01 \mathrm{M}$, sodium citrate buffer, pH6.0, for antigenic retrieval. Later, the sections were blocked with normal non-immume serum for $20 \mathrm{~min}$ and then incubated by mouse anti-Ki-67(Maixin, China) overnight at $4{ }^{\circ} \mathrm{C}$. The next day, the sections were treated with secondary antibody followed by streptavidin-horseradish peroxidase complex. Finally, DAB was used for colour development. PBS was used to replace the primary antibody as the negative control. Finally, the stained slides were evaluated independently by two pathologists who were blind to the clinical parameters. The positive tumor cells were stained for $\mathrm{Ki}-67$ protein in the nucleus.

\section{Plasmid construction, transfection and luciferase assays} The miR-384 binding site in the AKT3 is located at 4362-4367 bp, whose full length of $3^{\prime}$ UTR is $5439 \mathrm{bp}$. The region of human AKT3 3'UTR at 4141-4510 was PCR-amplified and inverted into the XhoI/NotI sites of the psiCHECK-2 luciferase reporter plasmid (Promega). The primers were as follows: F: CCGCTCGAG ATACACGCAAATACACTCC; R: GGGGCGGCC GCCTTCTACAGTATCCACCAC. Cells were seeded in 24 -well plates $\left(1 \times 10^{5} /\right.$ well $)$ the day before transfection. Then the psiCHECK-2-luciferase reporter gene plasmids psiCHECK-2-AKT3-3'-UTR or control-luciferase plasmid were transfected into the cells with the control pRL-TK Renilla plasmid (Promega) by Lipofectamine 2000 Reagent (Invitrogen). Luciferase and Renilla activities were assayed $48 \mathrm{~h}$ after transfection by the Dual Luciferase Reporter Assay Kit (Promega) following the manufacturer's instructions. All experiments were conducted at least 3 times and the data were presented as mean \pm standard deviation (mean $\pm \mathrm{SD}$ ).

\section{Statistical analysis}

All statistical analyses were carried out by SPSS20.0 for Windows. The data were expressed as the means \pm standard deviations (SD) from at least three independent experiments. The comparisons of the means were carried out by one-way analysis of variance (ANOVA) test with post hoc contrasts by LSD test. Comparisons were considered significant when $\mathrm{p}<0.05$. The comparison was conducted by Mann-Whitney U-test. Spearman's correlation analysis was carried out to analyze the relationship between miR-384 expression and AKT3 expression. Statistically significant data were indicated by asterisks: "p $<0.05$ or * $\mathrm{p}<0.01$.

\section{Results}

\section{MiR-384 was down-regulated in CRC tissues}

The expression of miR-384 was detected by qPCR analysis in 26 cases of CRC biopsies and their matched normal samples obtained from the Third affiliated Hospital. It was found that miR-384 was down-regulated in $88.5 \%$ $(23 / 26)$ of CRC tissue samples (T) compared to their matched adjacent normal tissues (N) (Fig. 1a). Student's $t$ test showed that the expression level of miR-384 in CRC tissues samples was significantly lower than that in adjacent normal tissues (Fig. 1b, c). Taken together, these results showed that miR-384 was down-regulated in CRC tissues.

\section{Over-expression of miR-384 inhibited the proliferation of CRC cells in vitro and in vivo}

To explore the possible role of miR-384 on the proliferation of CRC, MTT, soft agar and colony formation assay were performed with the stable cells of SW480/miR-384, SW480/Vector, HCT116/miR-384 and HCT116/Vector (Fig. 2a). The results showed that the over-expression of miR-384 obviously decreased the OD value of MTT, the colony number of soft agar and colony formation assay of SW480 and HCT116 (Fig. 2b-g). So, miR-384 overexpression significantly inhibited the proliferation of CRC cells in vitro. To further confirm the effect of miR-384 in inhibiting the proliferation of CRC cells in vivo, we performed the tumorigenesis assay in nude mice and found that the volume of tumors in SW480/miR-384 group was much smaller than that in SW480/Vector group (Fig. 2h). IHC confirmed that the tumors of the SW480/miR-384 group showed much lower Ki-67 indices than that in control group (Fig. 2i, j).

\section{Inhibition of endogenous miR-384 promoted the proliferation of CRC cells in vitro and in vivo}

The proliferative abilities of the stable cells LOVO/miR384-in, LOVO/NC, SW620/miR-384-in and SW620/NC were detected by MTT, soft agar and colony formation assay (Fig. 3a). The results demonstrated that the suppression of miR-384 obviously increased the OD value of MTT, the colony number of soft agar and colony formation assay of LOVO and SW620 cells compared with their negative control group (Fig. $3 \mathrm{~b}-\mathrm{g}$ ). Therefore, miR384 knock-down significantly increased the proliferative abilities of CRC cells in vitro. To further observe the inhibition effects of miR-384 on CRC proliferation in vivo, $\mathrm{LOVO} / \mathrm{miR}$-384-in cells and the control cells LOVO/NC were injected to the hind limbs of the nude mice. Results demonstrated that LOVO/miR-384-in group showed much lager tumors and much higher Ki-67 indices than that in LOVO/NC group (Fig. $3 \mathrm{~h}-\mathrm{j}$ ). 

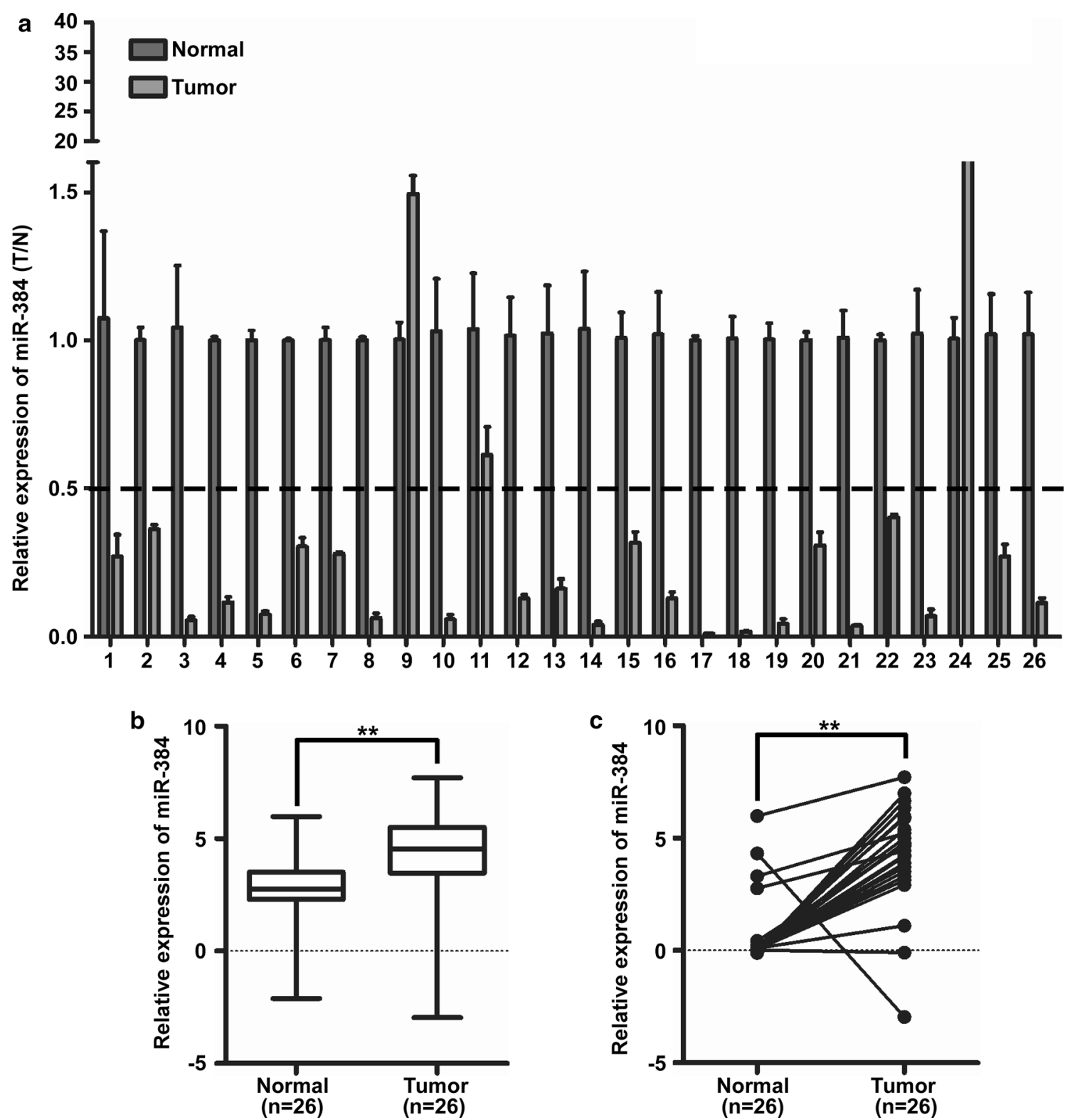

Fig. 1 MiR-384 was down-regulated in CRC tissues. a Expression of miR-384 in 26 cases of fresh human CRC tissues and their matched adjacent normal tissues by real-time PCR analysis; miR-384 expression was normalized to U6 and expressed relative to the matched adjacent normal tissues $\left(2^{-\Delta \Delta C \mathrm{~T}}\right)$. b, c Mean expression of miR-384 in 26 cases of fresh human CRC tissues and their matched adjacent normal tissues by real-time PCR ( $\triangle C_{T}$, $\mathrm{n}=26,{ }^{* *} p<0.01$ ), boundaries of boxes represent bounding of the boxes and stand for the lower and upper quartile. Lines within the boxes and whiskers represent median and extremum (maximum and minimum)

MiR-384 decreased AKT3 expression by directly binding to it's 3'UTR in CRC cells

The publicly available bioinformatic algorithms (TargetScan, miRada) were firstly used to analyze the target gene of miR-384. The results indicated that AKT3 was the theoretical target gens of miR-384 (Fig. 3a). Real-time PCR and western blot analyses showed that the mRNA and protein levels of AKT3 was significantly down-regulated in miR-384 over-expressed cells, whereas they were up-regulated with the inhibition of miR-384 (Fig. 4b, c). To confirm whether AKT3 was directly inhibited by miR-384, the dual-luciferase reporter system was performed. It was found that the co-expression of miR-384 markedly inhibited the Renilla luciferase reporter activity of the wild-type AKT3 3'UTR, but did not change the activity of the mutant 3 'UTR constructs and their scramble vectors (Fig. 4d). The above results verified that AKT3 was the target gene of miR-384. 

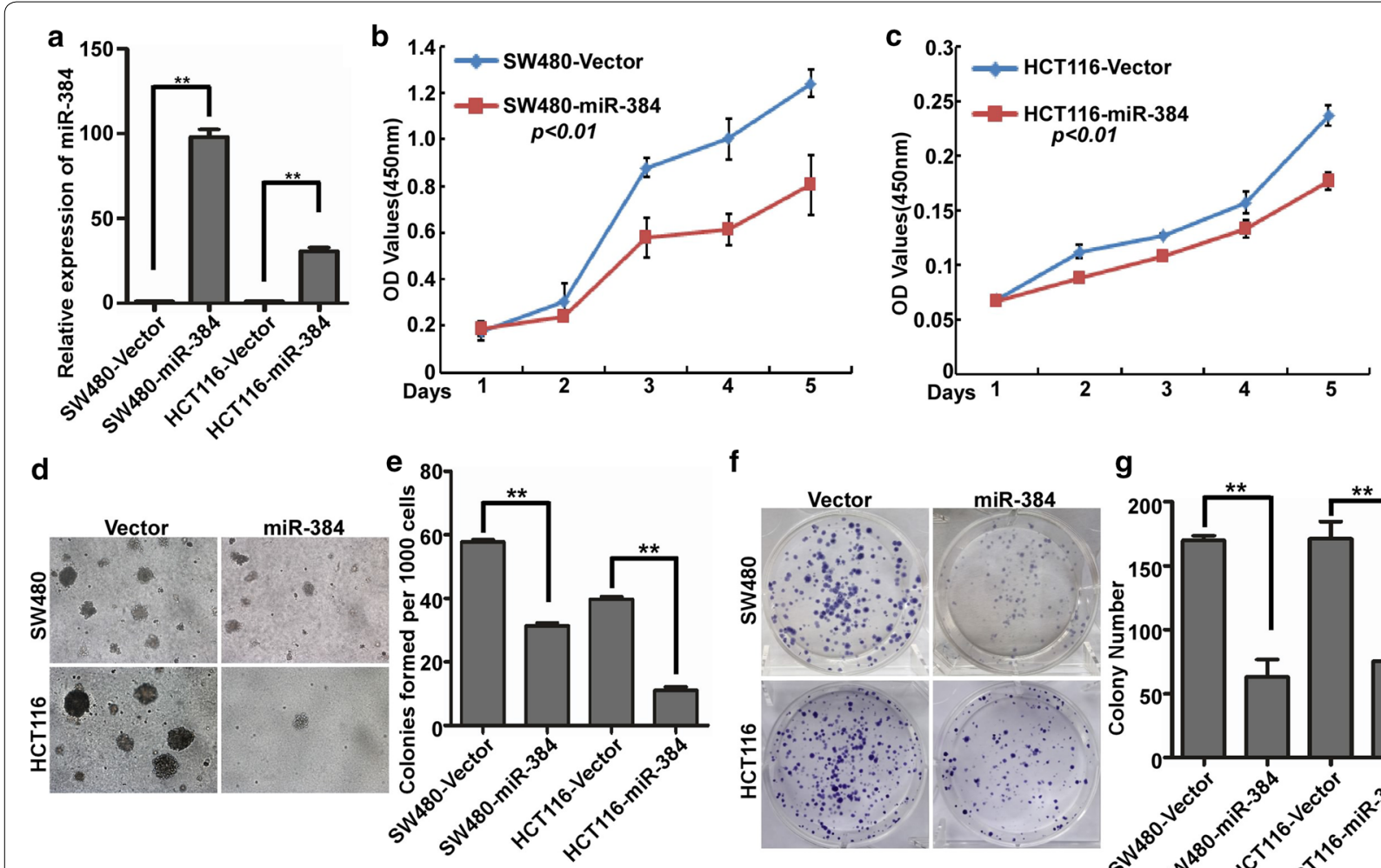

$\mathbf{f}$

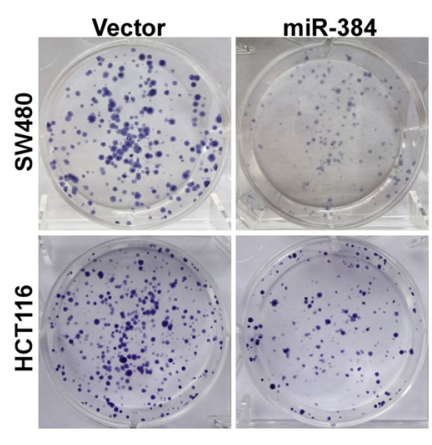

g

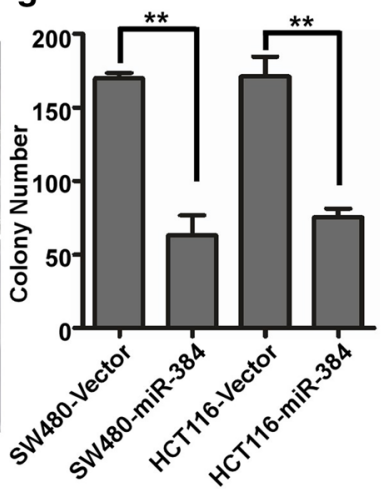

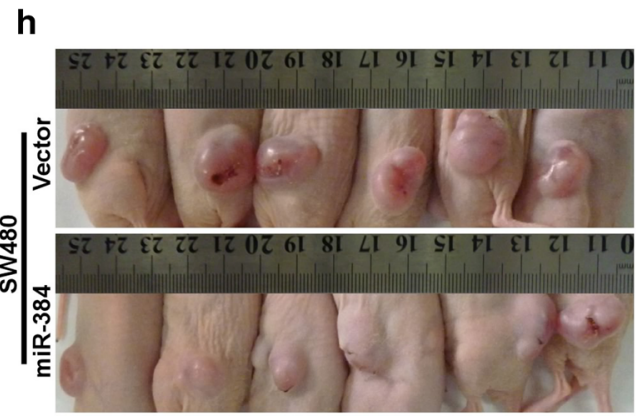
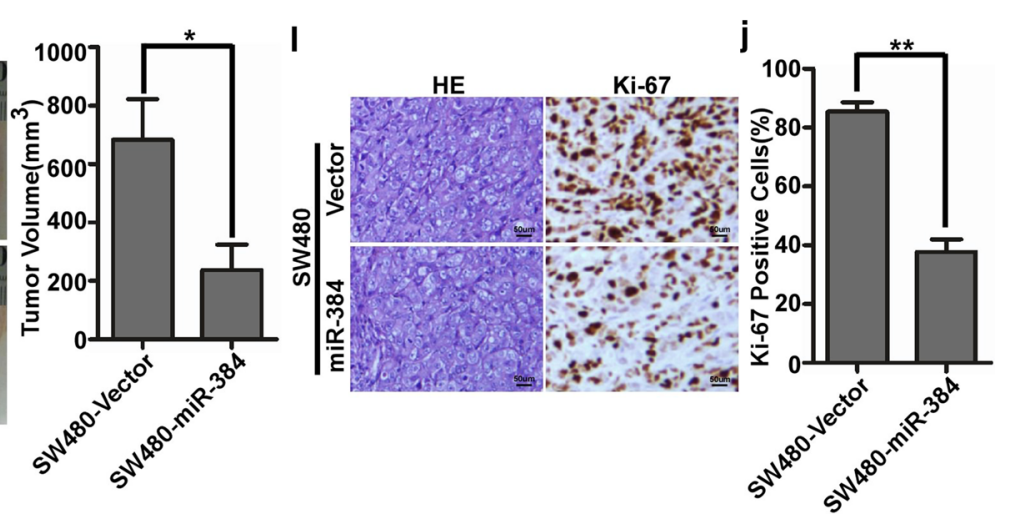

Fig. 2 Over-expression of miR-384 inhibited the proliferation of CRC cells in vitro and in vivo. a Over-expression of miR-384 in SW480 and HCT116 CRC cells verified by real-time PCR. $\mathbf{b}-\mathbf{g}$ The proliferative ability of the indicated cells detected by MTT assays, colony formation assays and soft agar assays. Only cell colonies containing more than 50 cells were counted. Error bars represent mean \pm SD from 3 independent experiments. ${ }^{* *} p<0.01$. h SW480/miR-384 and SW480/Vector cells were injected into the hind limbs of nude mice $(n=6)$. The tumor volume data were presented as the mean \pm SD. $\mathbf{i}, \mathbf{j}$ Histopathological analyses of xenograft tumours. The tumor sections were stained with H\&E or subjected to IHC staining using an antibody against Ki-67. Error bars represent mean \pm SD from three independent experiments. ${ }^{* *} p<0.01$

\section{Restoration the expression of AKT3 played an important} role in miR-384-inhibited proliferation of CRC

To further confirm the role of miR-384 in the progression of CRC, we next restored the expression of AKT3 in SW480/miR-384 cells (Fig. 5c) by transfection of AKT3 ORF constructs without $3^{\prime}$ UTRs, and observed their effects on proliferation. The results showed that the proliferative abilities of SW480/miR384 cells increased with the restoration of AKT3 both in vitro (Fig. $5 \mathrm{~d}-\mathrm{f}$ ). The in vivo tumorgenesis assay in nude mice showed the same trend (Additional file 1: Figure S1A, B). The above results confirmed that miR-384 inhibit the proliferation of CRC by targeting AKT3. 

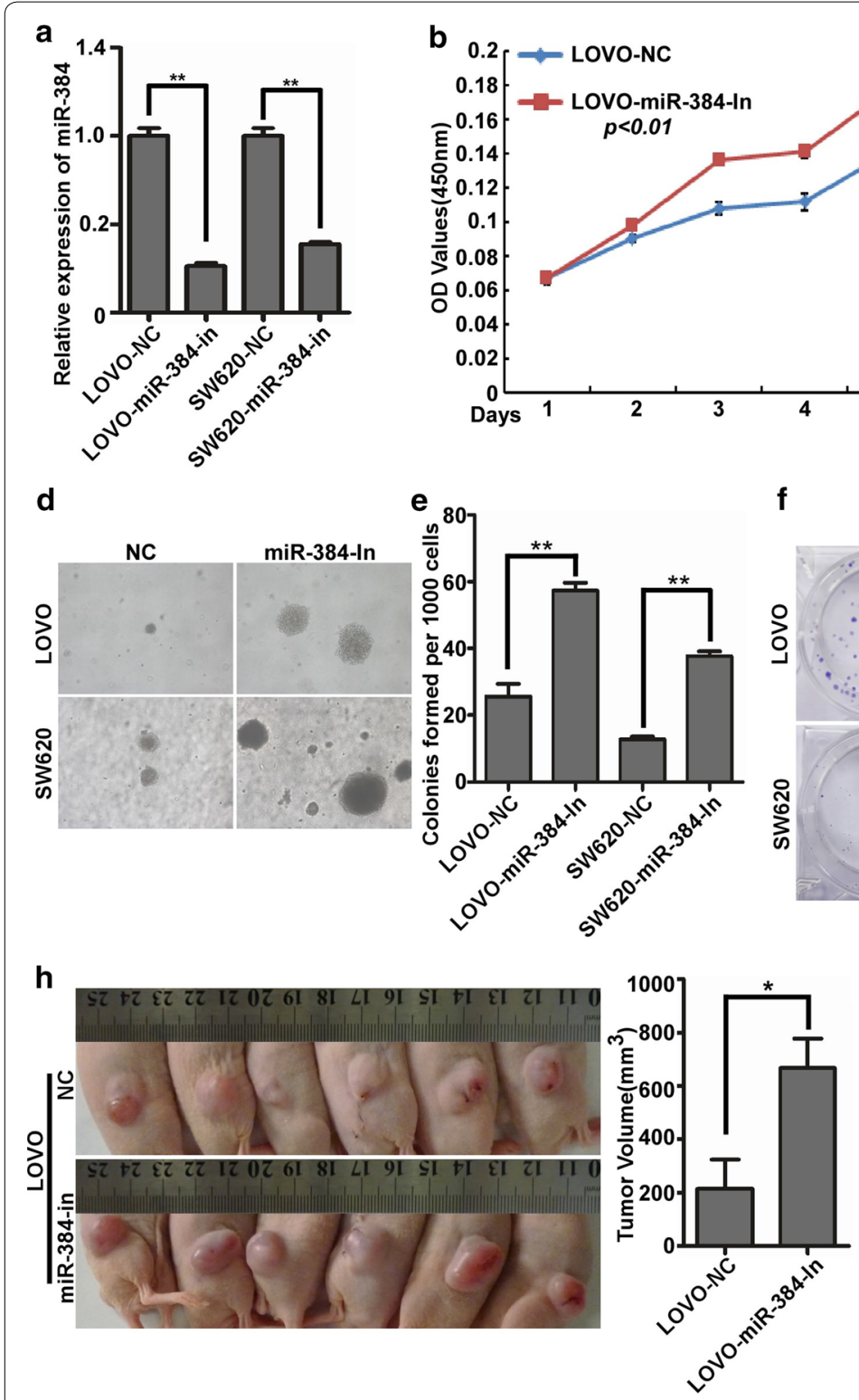

c

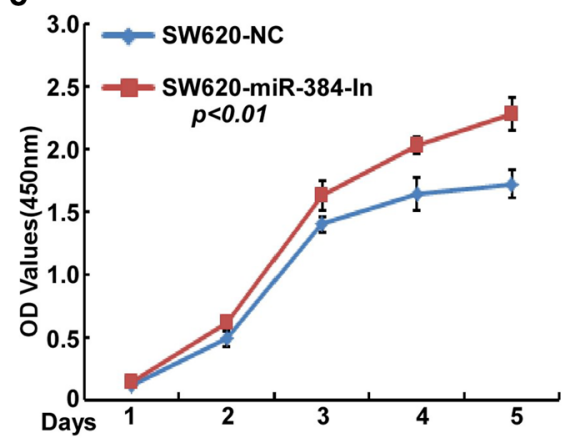

f

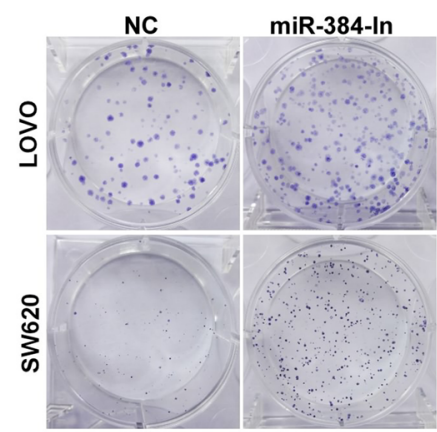

g

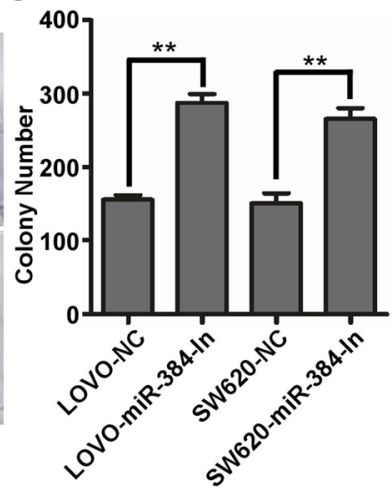

i

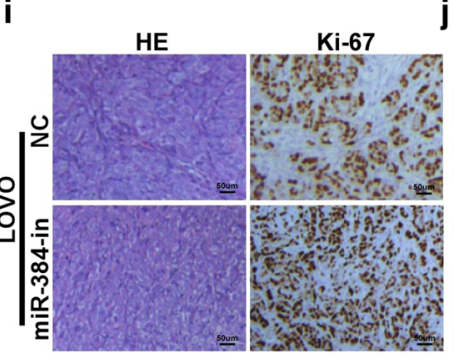

j

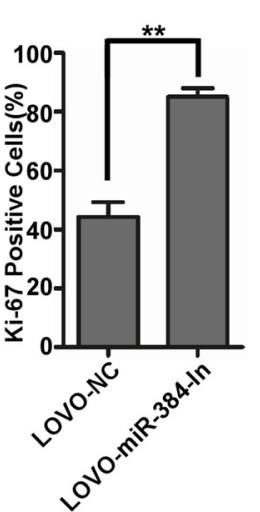

Fig. 3 Inhibition of endogenous miR-384 promoted the proliferation of CRC cells in vitro and in vivo. a Expression of miR-384 in LOVO and SW620 CRC cells transfected with inhibitor or their paired negative control lentiviral vector was detected by real-time PCR. $\mathbf{b}-\mathbf{g}$ The proliferative ability of the indicated cells detected by MTT assays, colony formation assays and soft agar assays. Only cell colonies containing more than 50 cells were counted. Error bars represent mean \pm SD from 3 independent experiments. ${ }^{* *} \mathrm{p}<0.01$. e LOVO/miR-384-in and LOVO/NC cells were injected into the hind limbs of nude mice $(n=6)$. The tumor volume data were presented as the mean $\pm S D$. $\mathbf{i}, \mathbf{j}$ Histopathological analyses of xenograft tumours. The tumor sections were stained with H\&E or subjected to IHC staining using an antibody against Ki-67. Error bars represent mean \pm SD from three independent experiments. ${ }^{* *} \mathrm{p}<0.01$

The expression of miR-384 was negatively correlated with the expressions of AKT3 in CRC tissues

We detected the expression of AKT3 in 10 cases of CRC and their paired normal colorectal tissues and the data were normalized to 2 housekeeping genes (GAPDH and $\beta$-actin). The standard deviations normalized to GAPDH was lower than that of $\beta$-actin (Additional file 1: Figure S2A). Therefore, we used GAPDH for normalization of AKT3 expression analysis to further verify the correlation between the expression of miR-384 and AKT3 was analyzed in another 10 freshly collected CRC biopsies and paired normal tissues. It was showed that miR-384 


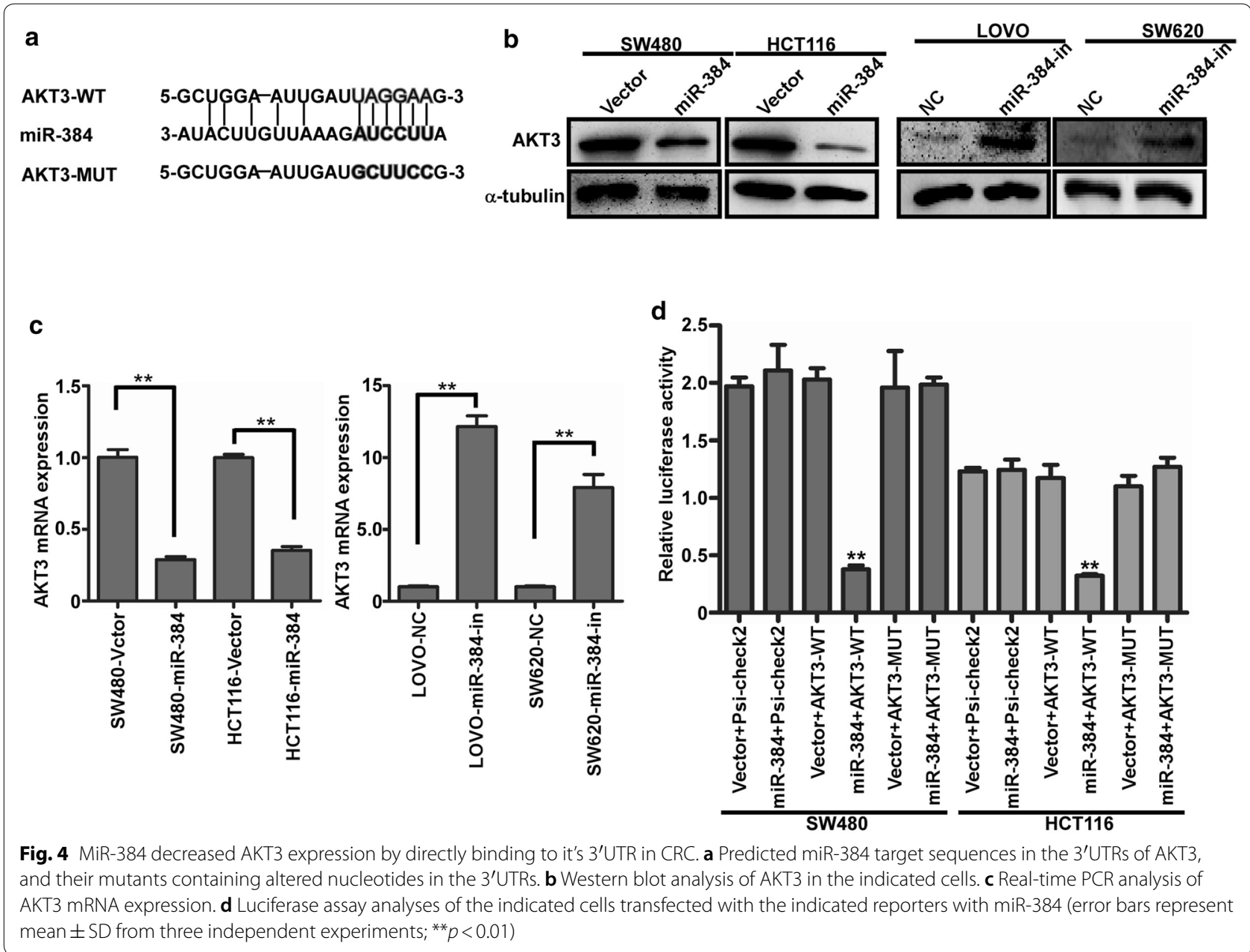

was down-regulated in CRC tissues while AKT3 was upregulated in CRC tissues (Fig. 6a). Spearman correlation analyses demonstrated that the expression of miR-384 expression was negatively correlated with the expressions of AKT3 (Fig. 6b).

\section{Discussion}

MiRNAs are small regulatory molecules that negatively regulate their target gene by directly binding their mRNAs [17]. These small non-coding RNA molecules could function as oncogenes or tumor suppressors [18]. Accumulating evidences have verified that the dysregulation of miRNAs is closely related to the development and progression of cancer [19-22]. Recent studies have demonstrated that miRNAs play essential roles in the initiation and progression of CRC in recent studies [23, 24]. Up to now, the deregulation of miR-384 has only been observed in a few tumor types, suggesting its function as a cancer suppressor gene. For instance, a microarray showed that miR-384 was down-regulated in laryngeal carcinoma [25]. Another research implies mir-384 might play an important role in metastasis of melanoma by binding to the $3^{\prime} \mathrm{UTR}$ of HDAC3 [26]. Recently, it was reported that miR-384 exerted tumor-suppressive functions by binding to the $3^{\prime}$ UTR of PIWIL4 in glioma [27]. However, it was not clear whether the dysregulation of miR-384 was associated with the proliferation of CRC. In our previous study, it has shown that with the restoration of KRAS and CDC42, the number of metastatic nodules in SW480 cells with miR-384 over-expression restored, too. But the volume of metastatic nodules could not be restored accordingly. This result suggested miR-384 might regulate the proliferation of CRC. Therefore, we focused on delineating the role and mechanism of miR384 in CRC proliferation in the current study.

To explore the function of miR-384 in the proliferation of CRC, MTT, colony formation and soft agar assays were conducted. The results showed that the growth and proliferation of CRC cells were obviously inhibited by miR-384 over-expression in vitro. Moreover, the results 


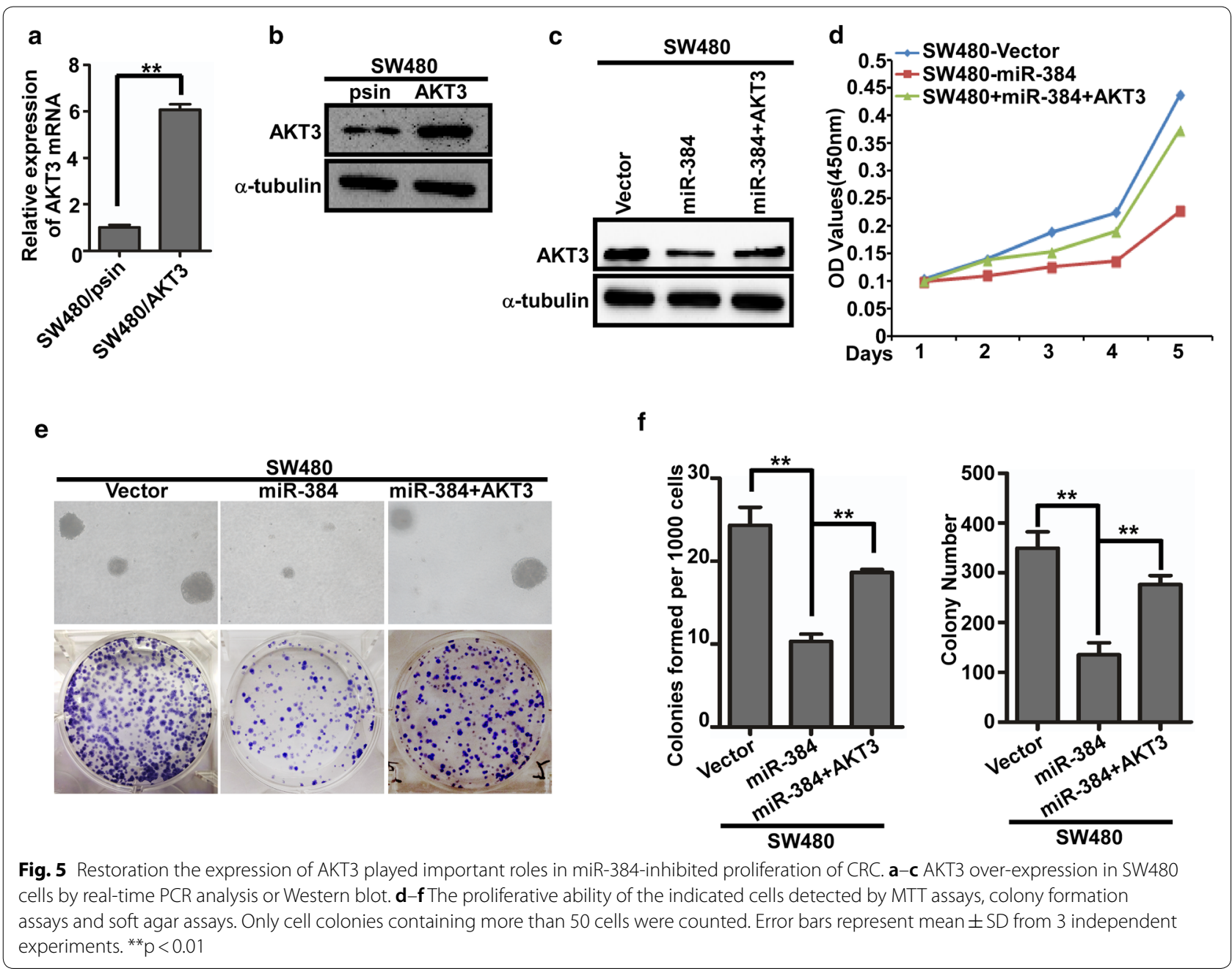

of the tumourigenesis assay in nude mice verified that miR-384 significantly promoted the growth of CRC cells in vivo. Moreover, the inhibition of miR-384 significantly promoted the proliferation in vitro and the ability of tumor formation in vivo of the CRC cells. In conclusion, the results demonstrated that miR-384 could inhibit the proliferation of CRC. Therefore, it is crucial to elucidate the molecular mechanism underlying the inhibition role of miR-384 in CRC proliferation, which would contribute to providing potential therapeutic targets for CRC.

As we know, miRNAs functions through regulating their target genes by cleavaging their mRNA and inhibiting the synthesis of the protein. In this study, we selected AKT3 as a target gene of miR-384 via the publicly available bioinformatic algorithms analysis. AKT3 has been reported to be involved in cancer progression and function as an oncogene in many types of cancers by regulating the PIK3/AKT signal pathway [28-30].
Recently, it was found that the expression of AKT3 was upregulated in thyroid cancer and that the inhibition of AKT3 inhibited it's proliferation [31]. In this study, we detected the expression of AKT3 in CRC and found that it was significantly upregulated. Notably, AKT3 has been found to be regulated by several miRNAs, such as miR-29a, miR-338-3p and miR-145 [32-34]. To further verify whether AKT3 was a target gene of miR-384 in $\mathrm{CRC}$, luciferase reporter assay, qRT-PCR, and western blot were conducted. The results confirmed that AKT3 was a new target of miR-384. In addition, we found that the expression of AKT3 was upregulated in CRC tissues and was inversely correlated with miR-384 expression. Furthermore, it was found that the importance of AKT3 in mediating the effect of miR-384 was substantiated by the finding that AKT3 overexpression rescued the miR384-mediated inhibitory effect on CRC cells. 

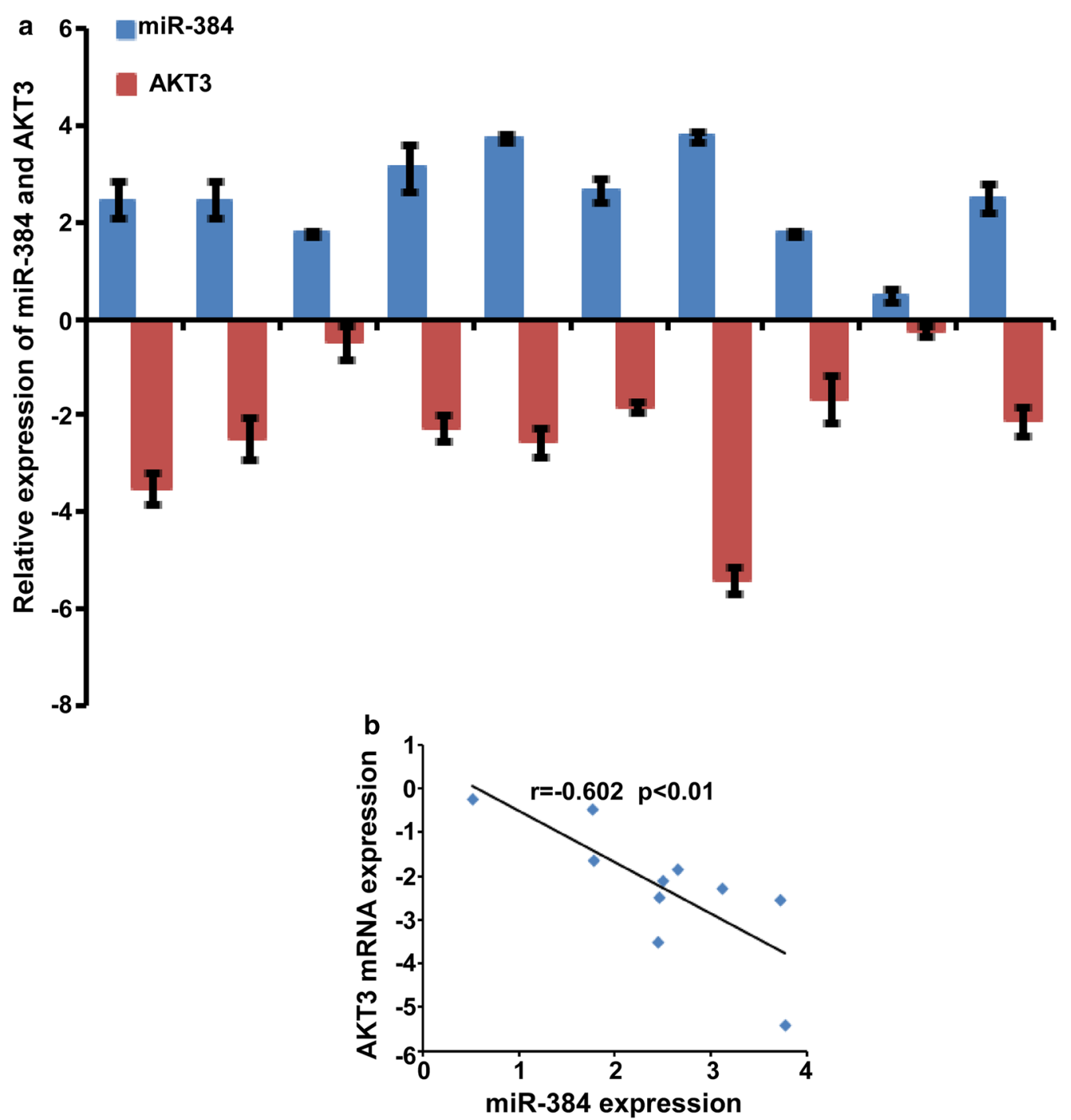

Fig. 6 Restoration the expression of AKT3 played important roles in miR-384-inhibited proliferation of CRC. a The expression of miR-384 and AKT3 were detected by real-time PCR $\left(\Delta C_{T}, n=10\right)$. b Spearman correlation analyses of miR-384 expression and AKT3 mRNA expression

\section{Conclusion}

In brief, our findings confirmed that miR-384 suppressed the proliferation of CRC by directly targeting AKT3. Identification of the miR-384/AKT3 axis in CRC proliferation would contribute to better understanding of the molecular mechanisms underlying CRC and provide potential diagnostic and prognostic biomarker for CRC.

\section{Additional file}

Additional file 1. Additional table and figures.

\section{Abbreviations}

CRC: colorectal cancer; miR-384: microRNA 384; AKT3: AKT serine/threonine kinase 3; RT: reverse transcription; RT-qPCR: real time quantitative polymerase chain reaction; MTT: 3-(4,5-dimethylthiazol-z-yl)-2,5-diphenyltetrazolium bromide; DMSO: dimethyl sulphoxide; PVDF: polyvinylidene difluoride.

\section{Authors' contributions}

YHH designed the experiments; WYX conducted experiments; HFZ provided research materials and methods; $Z Y Z$ analyzed data; $Y X W$ and FR wrote the manuscript. All authors read and approved the final manuscript.

\section{Acknowledgements}

We'd like to express our sincere thanks to all those who have lent us hands in the improvements to the English language within our revised manuscript.

\section{Competing interests}

The authors declare that they have no competing interests. 


\section{Availability of data and materials}

We declared that materials described in the manuscript, including all relevant raw data, will be freely available to any scientist wishing to use them for noncommercial purposes, without breaching participant confidentiality.

\section{Consent for publication}

The informed consent of patients was waived because the patients were all unconscious and the study did not involve additional procedures to the standardized clinical protocols, apart form the anonymous treatment of data.

\section{Ethics approval and consent to participate}

Approval was obtained from the Third Affiliated Hospital, Xinxiang Medical University. (Xinxing, China) for the use of clinical materials for research purposes. All samples were collected and analyzed with the prior written, informed consent of the patients, which were ethics approval and consent.

\section{Funding}

This work was supported by the National Natural Science Foundation of China (81702850) and grants from the Scientific Research Fund of Xinxiang Medical University (505290).

\section{Publisher's Note}

Springer Nature remains neutral with regard to jurisdictional claims in published maps and institutional affiliations.

\section{Received: 10 July 2018 Accepted: 30 August 2018} Published online: 03 September 2018

\section{References}

1. Chen W, Zheng R, Baade PD, Zhang S, Zeng H, Bray F, Jemal A Yu XQ, He J. Cancer statistics in China, 2015. CA Cancer J Clin. 2014:66(2):115-32.

2. Liu S, Zheng R, Zhang M, Zhang S, Sun X, Chen W. Incidence and mortality of colorectal cancer in China, 2011. Chin J Cancer Res. 2015;27(1):22-8.

3. Chen W, Zheng R, Zeng H, Zhang S, He J. Annual report on status of cancer in China, 2011. Chin J Cancer Res. 2015;27(1):2-12.

4. Harris TJ, McCormick F. The molecular pathology of cancer. Nat Rev Clin Oncol. 2010;7(5):251-65.

5. Ladabaum U, Clarke CA, Press DJ, Mannalithara A, Myer PA, Cheng I, Gomez SL. Colorectal cancer incidence in Asian populations in California: effect of nativity and neighborhood-level factors. Am J Gastroenterol. 2014;109(4):579-88.

6. Ogino S, Lochhead P, Giovannucci E, Meyerhardt JA, Fuchs CS, Chan AT Discovery of colorectal cancer PIK3CA mutation as potential predictive biomarker: power and promise of molecular pathological epidemiology. Oncogene. 2014;33(23):2949-55.

7. Network Cancer Genome Atlas. Comprehensive molecular characterization of human colon and rectal cancer. Nature. 2012;487(7407):330-7.

8. Paulmurugan R. MicroRNAs - a new generation molecular targets for treating cellular diseases. Theranostics. 2013;3(12):927-9.

9. Zhang GJ, Li JS, Zhou H, Xiao HX, Li Y, Zhou T. MicroRNA-106b promotes colorectal cancer cell migration and invasion by directly targeting DLC1. J Exp Clin Cancer Res. 2015:34:73.

10. Kloosterman WP, Plasterk $\mathrm{RH}$. The diverse functions of micro-RNAs in animal development and disease. Dev Cell. 2006;11(4):441-5.

11. He L, Hannon GJ. MicroRNAs: small RNAs with a big role in gene regulation. Nat Rev Genet. 2004;5(7):522-31.

12. Bonfrate L, Altomare DF, Di Lena M, Travaglio E, Rotelli MT, De Luca A, Portincasa P. MicroRNA in colorectal cancer: new perspectives for diagnosis, prognosis and treatment. J Gastrointest Liver Dis. 2013;22(3):311-20.

13. Vychytilova-Faltejskova P, Merhautova J, Machackova T, GutierrezGarcia I, Garcia-Solano J, Radova L, Brchnelova D, Slaba K, Svoboda M Halamkova J, Demlova R, Kiss I, Vyzula R, Conesa-Zamora P, Slaby O. MiR-215-5p is a tumor suppressor in colorectal cancer targeting EGFR ligand epiregulin and its transcriptional inducer HOXB9. Oncogenesis. 2017;6(11):399.

14. Sui GQ, Fei D, Guo F, Zhen X, Luo Q, Yin S, Wang H. MicroRNA-338-3p inhibits thyroid cancer progression through targeting AKT3. Am J Cancer Res. 2017;7(5):1177-87.

15. Wang G, Pan J, Zhang L, Wei Y, Wang C. Long non-coding RNA CRNDE sponges miR-384 to promote proliferation and metastasis of pancreatic cancer cells through upregulating IRS1. Cell Prolif. 2017:50(6):e12389.

16. Wang YX, Chen YR, Liu SS, Ye YP, Jiao HL, Wang SY, Xiao ZY, Wei WT, Qiu JF, Liang L, Liao WT, Ding YQ. MiR-384 inhibits human colorectal cancer metastasis by targeting KRAS and CDC42. Oncotarget. 2016:7(51):84826-38.

17. Ambros $V$. The functions of animal microRNAs. Nature. 2004;431(7006):350-5.

18. Shenouda SK, Alahari SK. MicroRNA function in cancer: oncogene or a tumor suppressor? Cancer Metastasis Rev. 2009;28(3-4):369-78.

19. Iorio MV, Croce CM. MicroRNA dysregulation in cancer: diagnostics, monitoring, and therapeutics. A comprehensive review. EMBO Mol Med. 2017;9(6):852

20. Pencheva N, Tavazoie SF. Control of metastatic progression by microRNA regulatory networks. Nat Cell Biol. 2013;15(6):546-54.

21. Pencheva N, Tran H, Buss C, Huh D, Drobnjak M, Busam K, Tavazoie SF. Convergent multi-miRNA targeting of ApoE drives LRP1/LRP8-dependent melanoma metastasis and angiogenesis. Cell. 2012;151(5):1068-82.

22. Mingozzi F, High KA. Therapeutic in vivo gene transfer for genetic disease using AAV: progress and challenges. Nat Rev Ge net. 2011;12(5):341-55

23. Sheng $L$, He P, Yang $X$, Zhou M, Feng Q. miR-612 negatively regulates colorectal cancer growth and metastasis by targeting AKT2. Cell Death Dis. 2015:6:1808

24. Ozawa T, Kandimalla R, Gao F, Nozawa H, Hata K, Nagata H, Okada S, Izumi D, Baba H, Fleshman J, Wang X, Watanabe T, Goel A. A microRNA signature associated with metastasis of T1 colorectal cancers to lymph nodes. Gastroenterology. 2018;154(4):844-8.

25. Tonouchi E, Gen Y, Muramatsu T, Muramatsu T, Hiramoto H, Tanimoto K, Inoue J, Inazawa J. miR-3140 suppresses tumor cell growth by targeting BRD4 via its coding sequence and downregulates the BRD4-NUT fusion oncoprotein. Sci Rep. 2018;8(1):4482

26. Zhang W, Qian S, Yang G, Zhu L, Zhou B, Wang J, Liu R, Yan Z, Qu X. MicroRNA-199 suppresses cell proliferation, migration and invasion by downregulating RGS17 in hepatocellular carcinoma. Gene. 2018;659:22-8

27. Eom S, Kim Y, Park D, Lee H, Lee YS, Choe J, Kim YM, Jeoung D. Histone deacetylase-3 mediates positive feedback relationship between anaphylaxis and tumor metastasis. J Biol Chem. 2014;289(17):12126-44.

28. Wang P, Fu T, Wang X, Zhu W. Primary, study of miRNA expression patterns in laryngeal carcinoma by microarray. Lin Chung Er Bi Yan Hou Tou Jing Wai Ke Za Zhi. 2010;24(12):535-8.

29. Zheng J, Liu X, Wang P, Xue Y, Ma J, Qu C, Liu Y. CRNDE promotes malig nant progression of glioma by attenuating miR-384/PIWIL4/STAT3 axis. Mol Ther. 2016;24(7):1199-215

30. Bellacosa A, Kumar CC, Di Cristofano A, Testa JR. Activation of AKT kinases in cancer: implications for therapeutic targeting. Adv Cancer Res. 2005;94:29-86.

31. Paul-Samojedny M, Pudelko A, Kowalczyk M, Fila-Danilow A, SuchanekRaif R, Borkowska P, Kowalski J. Combination therapy with AKT3 and PI3KCA siRNA enhances the antitumor effect of temozolomide and carmustine in T98G glioblastoma multiforme cells. BioDrugs. 2016:30(2):129-44

32. Grottke A, Ewald F, Lange T, Norz D, Herzberger C, Bach J, Grabinski N, Graser L, Hoppner F, Nashan B, Schumacher U, Jucker M. Down regulation of AKT3 increases migration and metastasis in triple negative breast cancer cells by upregulating s100a4. PLOS ONE. 2016;11(1):0146370.

33. Li R, Liu J, Li Q, Chen G, Yu X. miR-29a suppresses growth and metastasis in papillary thyroid carcinoma by targeting AKT3. Tumour Biol. 2016;37(3):3987-96.

34. Boufraqech M, Zhang L, Jain M, Patel D, Ellis R, Xiong Y, He M, Nilubol N, Merino MJ, Kebebew E. miR-145 suppresses thyroid cancer growth and metastasis and targets AKT3. Endocr Relat Cancer. 2014;21(4):517-31. 